\section{A CASE OF MONILIASIS}

BY

F. C. ORMEROD, M.D., F.R.C.S.

Professor in Oto-rhino-laryngology, University of London

AND

I. FRIEDMANN, M.D., M.R.C.S., D.C.P.

Reader in Bacteriology, University of London

(From the Institute of Laryngology and Otology, London)

Warnings on the danger of the newer antibiotics (British Medical Journal, 1951) have referred to the occasional development of moniliasis. A case of moniliasis of the oropharynx which spread to the bronchi and has apparently developed in the course of intensive and extensive antibiotic treatment of maxillary sinusitis with penicillin, streptomycin, and " aureomycin" may therefore be of some interest.

\section{Case History}

An unmarried woman aged 49 was quite well until May, 1950 , when she had a bad cold which did not clear up. She was given penicillin by her own doctor and was later seen by Mr. P. E. Roland, to whom we are indebted for details of the earlier findings. He found both nares occluded by a fibrinous membrane. Repeated cultures grew a Staphylococcus aureus, at first sensitive to both penicillin and streptomycin. Systemic courses of both drugs caused temporary improvement only, and she remained under medical supervision, as malignancy had been suspected.

A biopsy taken in June, 1950, of some soft tissue from the posterior pharyngeal wall showed chronic inflammation and no evidence of malignancy. She was again admitted to hospital owing to an exacerbation of her condition in September, 1950. A penicillin-resistant Staph. aureus was now grown from the nose, and she was treated with aureomycin. She also received deep $x$-ray treatment. A biopsy of the nasopharynx showed oedematous and largely necrotic material, but no evidence of malignancy or tuberculosis. There was some clinical improvement.

In December, 1950, however, she suddenly developed an oedema of the left orbit. She was again put on aureomycin and the left orbit was explored. No pus was found in the orbit or left frontal sinus, but the incision started to discharge. Penicillin, streptomycin, and heparin were given, in addition to the aureomycin she had been receiving. There was only little improvement in her condition, and for the first time yeast-like bodies were found in the purulent discharge. On February 23, 1951, she was admitted to the Royal National Throat, Nose and Ear Hospital in London.

Clinical Findings.- The patient was a frail woman weighing 7 st. ( $44.5 \mathrm{~kg}$.), as compared with $10 \mathrm{st} .6 \mathrm{lb} .(66.2 \mathrm{~kg}$.) a year before. Lesions resembling erythema multiforme lesions were found on the extremities. The lids of the left eye were inflamed and there was some periorbital oedema. Pus was discharging from the sinus above the internal angle of the eye. In the right naris there were adhesions between the septum and the middle turbinate. The left cavity was filled with pus and scabs; there was some ulceration of the nasal mucosa. Several ulcers $1-2 \mathrm{~cm}$. in diameter were seen on the soft palate, the uvula, and the back of the tongue. A radiograph of the chest on February 28 showed considerable consolidation of the right lower lobe. Bronchoscopy revealed a large quantity of fetid pus in the bronchi, and the right bronchus showed some sloughing. A great deal of purulent crumbling matter was aspirated for investigation. The patient's condition appeared to have improved after the bronchoscopy.

Treatment.-Penicillin and streptomycin were given. In view of the bacteriological findings the ulcers of the oral cavity were painted with gentian violet, and potassium iodide was given orally. This treatment seemed to have a beneficial effect.

Bacteriology.-Direct smears from the ulcers of the palate, from the nose, from stools, and, in particular, from the bronchoscopy aspirations showed crowds of yeast-like bodies and mycelia. Cultures yielded a profuse growth of Candida albicans. Fermentation tests:-Glucose: acid and gas. Maltose: acid and gas. Saccharose: nil. Lactose: nil.

Animal Inoculation.-Intravenous injection of $1 \mathrm{ml}$. of a saline suspension killed a rabbit (weight 2,000 g.) in three days. There were characteristic miliary abscesses in the kidneys and myocardium. Subcutaneous injection of $1 \mathrm{ml}$. of the above suspension produced a large subcutaneous abscess which healed spontaneously within a fortnight. Routine cultures from bronchial aspirations also grew penicillin-resistant Staph. aureus and streptomycin-sensitive Pseudomonas aeruginosa. Tubercle bacilli were not seen in the sputum. An autogenous vaccine of the $C$. albicans was being prepared, when the patient developed a severe haemoptysis and died on April 1.

\section{Summary of Post-mortem Findings}

Oral Cavity and Upper Respiratory Tract : Multiple ulcers of palate, uvula, back of the tongue, and nasal cavity. Suppuration in maxillary frontal and ethmoidal sinuses. Lungs : Extensive ulceration of right main bronchus, with perforation and haemorrhage mainly into the left lung. Diffuse bronchiectasis and suppurative bronchitis with confluent organizing bronchopneumonia of the right lung. Large penetrating abscess of the right lower lobe with organizing empyema. Intestinal Tract: No ulceration seen. Cultures from bronchi, ulcers of palate, nasopharynx, ethmoid, and intestines yielded a heavy growth of $C$. albicans. Penicillin-resistant Staph. aureus was grown from lungs.

The macroscopic appearance of the right lung was rather striking. The parenchyma was very firm and of greyish colour. The walls of the gaping dilated bronchi were greatly thickened and of an intensive white colour. Many larger bronchi were disintegrating, and one large branch led into an abscess which penetrated to the surface of the right lower lobe, causing a purulent pleurisy now partly organized.

However, we have been unable to find monilia in the sections of the lungs. There were masses of staphylococci in numerous bronchiectatic abscesses, but no yeast-like bodies or mycelia in the sections of the lung or sinuses stained with Gram or the periodic-acid-Schiff (P.A.S.) method. There were a few yeast-like bodies in sections of the ulcerated uvula.

\section{Discussion}

While it is always difficult to incriminate organisms which inhabit the human tissue as saprophytes it is recognized that under favourable-conditions they may become pathogenic (Davis et al., 1937). Treatment with the newer antibiotics chloramphenicol and aureomycin may prepare the ground for secondary mycotic invasion (Tomaszewski, 1951).

In our case the clinical picture and the repeated cultural findings of $C$. albicans are suggestive enough that a monilial infection has developed in the course of the treatment with various antibiotics, including several courses of aureomycin, leading to extensive ulceration of the oral cavity, the upper respiratory tract, and the right main bronchus. Diffuse bronchiectasis and suppurative bronchitis followed, with the development of staphylococcal bronchopneumonia.

We are indebted to Professor L. P. Garrod for his kind interest and advice.

\section{REFERENCES}

British Medical Journal, 1951, 1, 1196.

Davis, A. Hobson, Warren, Earl L., and Paterson, N. J. (1937). J. Lab. clin. Med., 22, 687.

Tomaszewski, T. (1951). British Medical Journal, 1, 388. 